\title{
Review Article \\ Cardiac injury and COVID-19: A Review on available present-day literature
}

\author{
Padma V. ${ }^{1}$, Rameez B. Raja ${ }^{2}$, Abhilash B. Nair ${ }^{3}$ \\ ${ }^{1}$ Professor, ${ }^{2}$ Assistant Professor, ${ }^{3}$ Junior Resident, Department of Medicine, Sree Balaji Medical College and Hospital, \\ Chennai, India
}

(Received: August $2020 \quad$ Revised: November $2020 \quad$ Accepted: November 2020)

Corresponding author: Abhilash B. Nair. Email: drabhilashnairb@gmail.com

\begin{abstract}
What started as a small cluster of viral pneumonia like cases in a province of China, in the early weeks of December, has blown out to be a full-fledged pandemic virtually affecting every continent. PubMed and Google search engines were used for literature search. The case fatality rate which is around $2.6 \%$ for corona virus disease (COVID-19) patient's rise to nearly 10.6\% in COVID-19 patients with prior cardio vascular disease. Common cardiac manifestations include heart failure and myocardial damage. Angiotensin converting enzyme (ACE2) plays an important part in the pathophysiology of heart function in hypertension and diabetes, two of the most common manifestations of heart failure and is the cellular entry point for the severe acute respiratory syndrome (SARS) virus. Patients with cardio vascular disease are believed to have increased secretion of ACE2 compared to healthy individuals and thus are at higher risk. Patients on medications with angiotensin converting enzyme inhibitor (ACEi) /angiotensin receptor blocker (ARB) have increased ACE2 levels and may be at an increased risk, however there is no statistically significant data to substantiate this claim.
\end{abstract}

Keywords: Heart failure; severe acute respiratory syndrome corona virus 2 (SARS-CoV-2); corona virus disease (COVID-19); Angiotensin converting enzyme (ACE2); injuries; angiotensin converting enzyme inhibitor (ACEi) /angiotensin receptor blocker (ARB)

Key points

1. Heart failure is defined as a complex clinical syndrome that results from any structural or functional impairment of ventricular filling or ejection of blood by the American heart association

2. COVID-19 is a pandemic caused by SARS-CoV-2 and very similar to bat corona virus.

3. ACE2 serve as a host for SARS-CoV-2. ACE2 is highly expressed in pericytes of adult human heart.

4. Patients with heart failure exhibited increased expression of ACE2 and thus are at risk of cardiac manifestation and injury

5. Among the cardiac patients, heart failure, atrial fibrillation (AF), coronary artery disease (CAD), and chronic kidney disease (CKD) were present in $40,36,30$, and $28 \%$ of the patients, respectively.

6. Case fatality rate was elevated among those with pre-existing comorbid conditions $-10.5 \%$ for cardiovascular disease, $7.3 \%$ for diabetes, $6.3 \%$ for chronic respiratory disease and $6.0 \%$ for hypertension.

7. Heart failure was the most common cause of death after acute respiratory distress syndrome (ARDS) and sepsis.

8. Creatine kinase (CK), CK-MB, lactate dehydrogenase, aspartate transferase, and erythrocyte sedimentation rate were significantly associated with the severity of hypokalaemia.

9. Elevated troponin I, NT-proBNP, hs-CRP correlate with severity of disease.

10. ACE2 is upregulated in patients with cardiovascular co morbidities like diabetes and hypertension, and patients on treatment with ACE inhibitors or ARB.

11. There is no urgent need to discontinue ACEIs or ARBs in COVID-19 patients.

\section{INTRODUCTION}

$\mathrm{H}$ eart diseases have been documented in "Die Cellularpathologie" in 1858 by Rudolf Virchow (1). Heart failure is defined as a complex clinical syndrome that results from any structural or functional impairment of ventricular filling or ejection of blood by the American Heart Association. This in turn leads to classical symptoms and signs of heart failure i.e. dyspnoea, fatigue, oedema, rales respectively. It is further classified into (i) Heart failure with reduced ejection fraction (HFrEF), defined as an ejection fraction $\leq 40 \%$, whereas (ii) Heart failure with preserved ejection fraction (HFpEF) is defined as an ejection fraction $\geq 50 \%$. Patients with an ejection fraction between this range are considered to have borderline HFpEF. Coronary artery disease, hypertension and diabetes mellitus are the most common risk factors associated with heart failure (2).

In the later weeks of December 2019, a surge of viral pneumonia cases was seen in Wuhan province of china. Gradually over a period of six months it spread to all continents. It was a beta corona virus with enveloped non segmented positive sense RNA. It has been named as Severe Acute Respiratory Syndrome Corona virus 2 (SARS-CoV-2) viruses by the International Taxonomy group. It is called Corona virus Disease 2019(COVID-19) by the World Health Organization (WHO). Earlier epidemics of corona virus include the severe acute respiratory syndrome corona virus (SARS-CoV) (3)) from China and Middle East Respiratory Syndrome corona virus (MERS-CoV) (4) from Saudi Arabia. SARS-CoV-2 shares about $80 \%$ of its genomic identity with that of SARS-CoV and is about $96 \%$ identical to the bat 
corona virus Bat-CoV RaTG13 (5). It is one of the largest viruses with a size from 27-34 kilobase. It is a zoonotic infection and affects the respiratory system more commonly in humans.

\section{Epidemiology}

Several cases of probable pneumonia were reported from Wuhan, China in the early weeks of December 2019. It was declared a public health emergency of international concern on January 30th, and a pandemic on $11^{\text {th }}$ March 2020. WHO report of $16^{\text {th }}$ August 2020(Situation Report 209) confirmed 21294845 cases with 761779 death, from nearly 213 countries (6). The number of cases in the 10 most affected countries (with approximate number of cases) are USA (5258565), Brazil (3275520), India
(2589682), Russia (922853), South Africa (583653), Peru (516296), Mexico (511369), Colombia (445111), Chile (383902) and Spain (342813), as per Corona virus (COVID-19) situation Report 209 (6).

\section{Clinical presentation}

SARS-CoV-2 predominantly spreads through respiratory droplets, but can spread through aerosols and stools as well. Viral transmission may occur from symptomatic as well as asymptomatic patients. Secondary infection rates range from $0.5 \%$ to $5 \%$. Reproductive number (R0) for the virus is around 2.2(7). Median incubation period is 4 to 5 days. Common symptoms include fever, dry cough, and myalgia, fatigue and diarrhoea (7; Chart1).

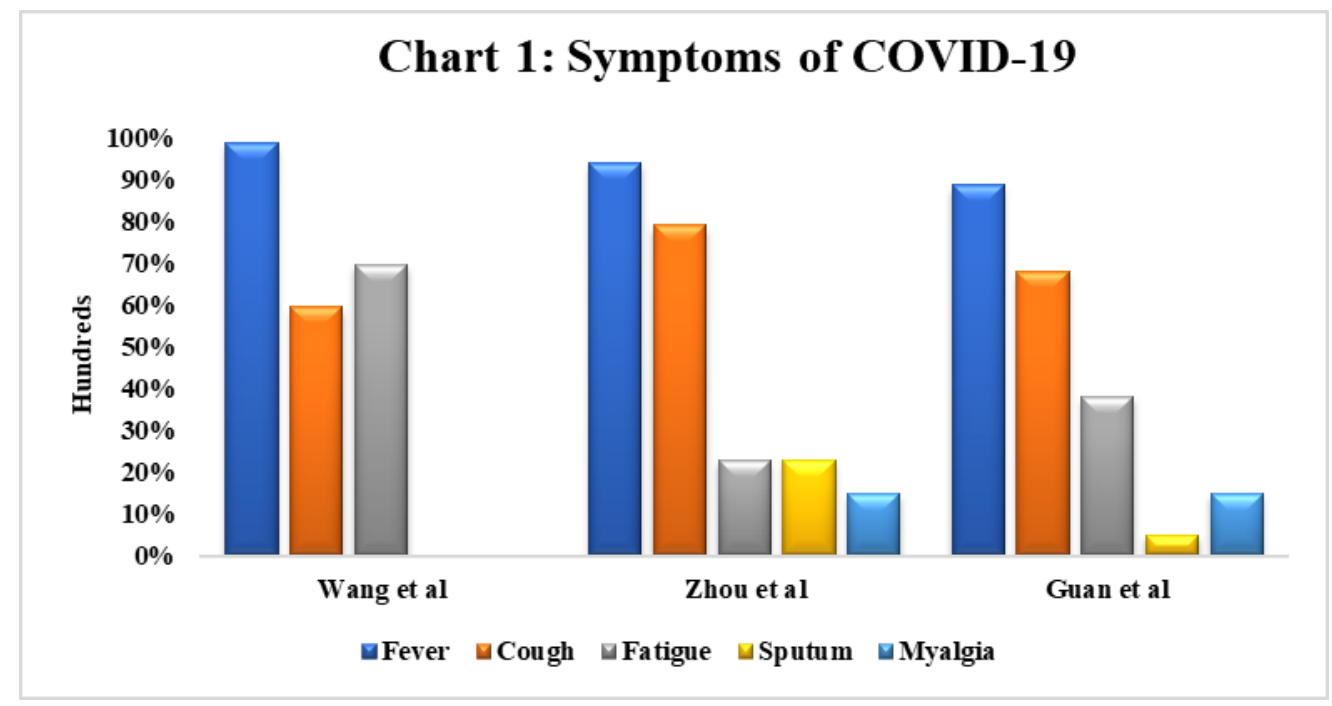

\section{Risk factors and COVID-19}

COVID-19 has been found to be associated with respiratory symptoms predominantly, however it has now been seen that it involves multiple organs including the heart and can present with nonrespiratory symptoms (8-13). Studies by $\mathrm{Wu}$ et al., Yang et al., and Arentz et al., have showed that patients with cardiovascular co-morbidities are at a higher risk for severe infection (8-10). Mehra et al., have shown that underlying cardiovascular disease is associated with an increased risk of in-hospital death among patients hospitalized with COVID-19(14) (Chart 2). Refractory COVID-19 patients had more underlying co-morbidities like diabetes and cardiovascular disease (15).

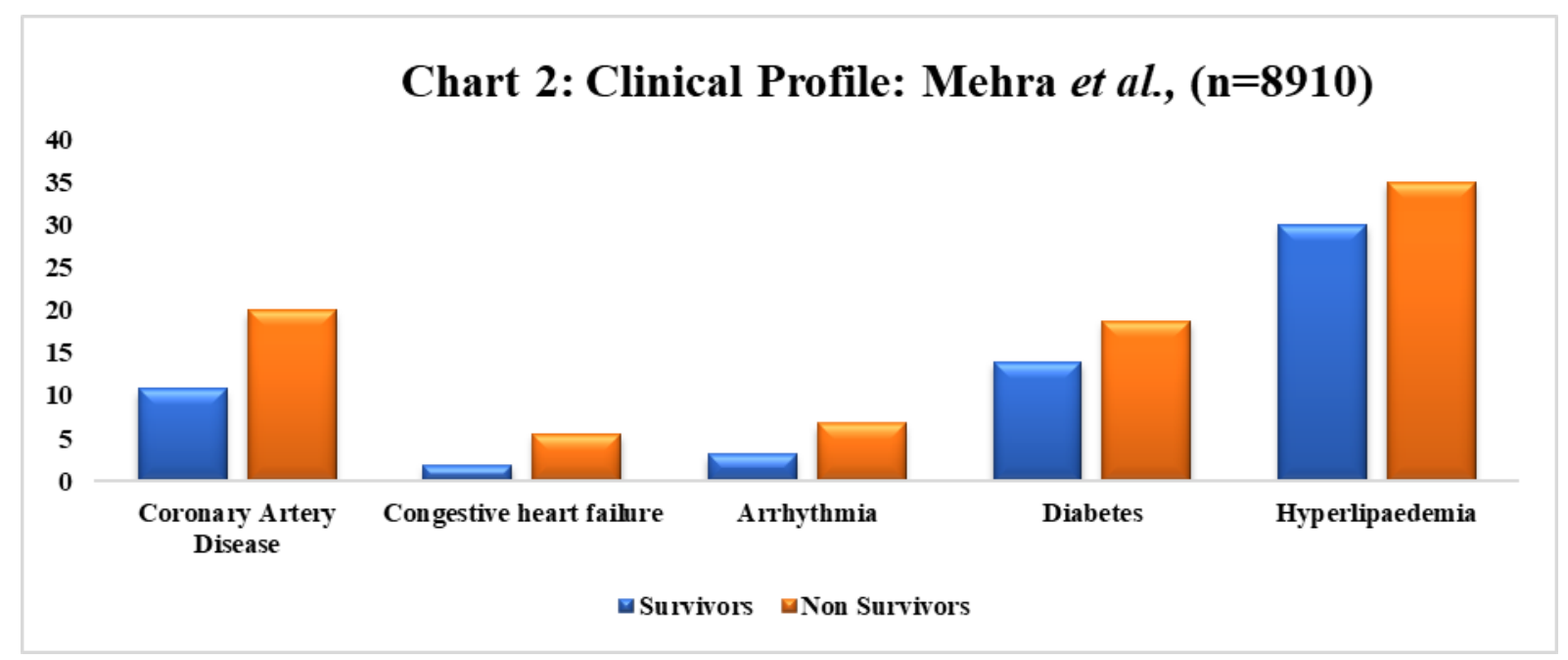

Among the cardiac patients, heart failure, atrial fibrillation $(\mathrm{AF})$, coronary artery disease (CAD), and CKD were present in $40,36,30$, and $28 \%$ of the patients (Chart 3). A comparison between different studies is shown in chart 4. Case fatality rate of patients with cardio vascular complications 
was $10.5 \%$ for cardiovascular disease, $7.3 \%$ for diabetes, $6.3 \%$ for chronic respiratory disease and $6.0 \%$ for hypertension as per data obtained from the Chinese centre for disease control and prevention $(16,17)$.

Women have stronger innate and adaptive immunity and greater resistance to viral infections than men as has been seen in the previous infection by SARS CoV-1(17). Thus, they are believed to survive the epidemic for longer than men.
Studies by Arentz et al., (12) showed that heart failure was present in $43 \%$ of cases in intensive care unit (ICU). An important observation done by Chaomin Win et al showed that the incidence of hypertension (27.4 vs 13.7), diabetes (16 vs 6 ) and cardiac disorder (5 vs 3 ) was higher in patients with ARDS vs non ARDS patients, due to COVID-19. Among the patients having ARDS, incidence of hypertension (17.5 vs 36.4 ), diabetes (12.5 vs 25 ) and cardiac disease (2.5 vs 9.1) among the survivor's versus non-survivors (18).
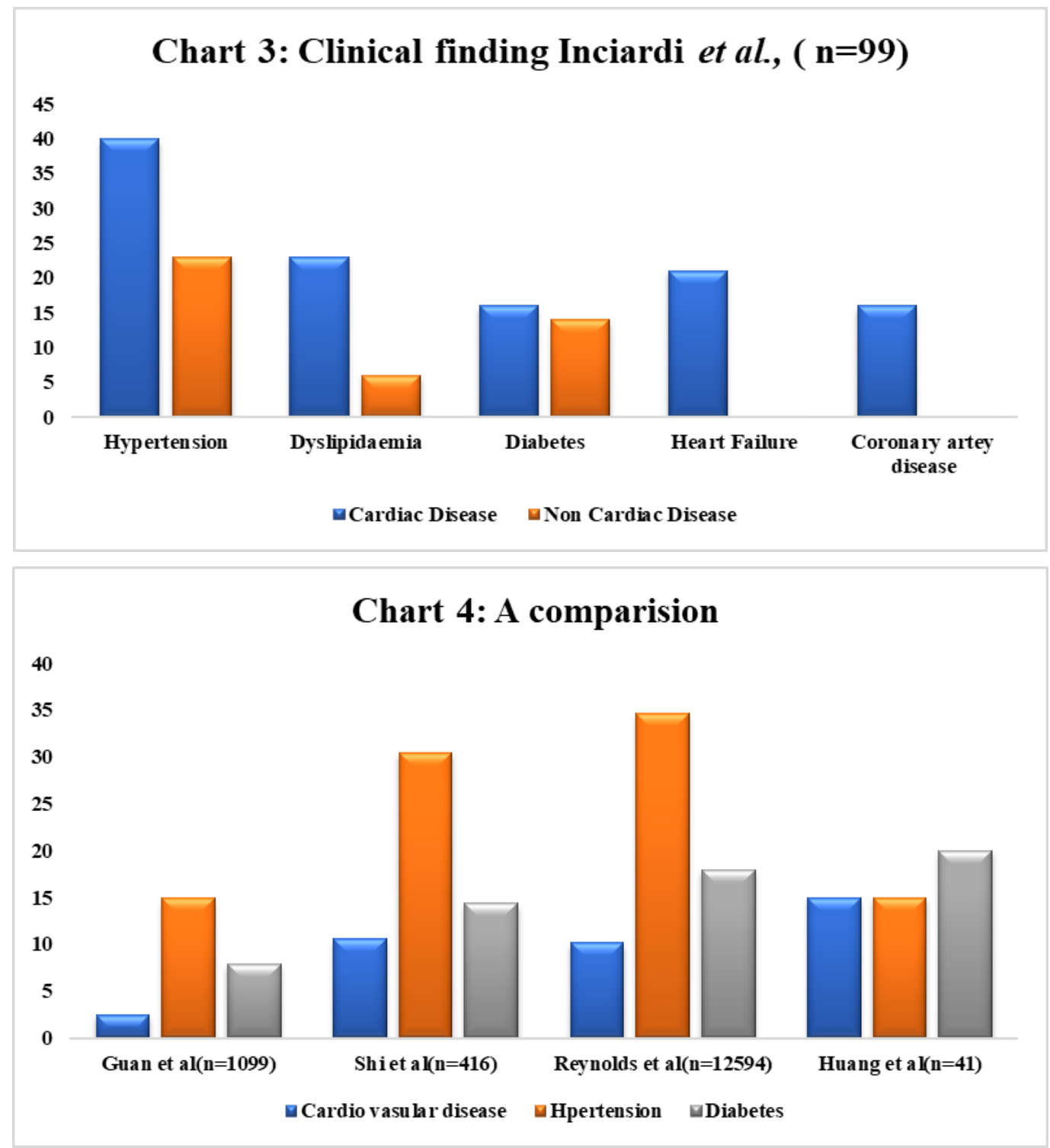

\section{Patho-physiology of myocardial injury}

Myocardial injury presentation may be asymptomatic or symptomatic (characterized by positive troponin levels). Troponin levels above the $99^{\text {th }}$ percentile upper reference limit is considered to be positive. Higher levels are seen in patients of COVID-19 especially patients who are critically ill (Chart 5). Hung et al showed that highly sensitive cardiac troponin I was elevated in $4 / 13$ of the ICU patients and $1 / 28$ in the non-ICU patients. The levels increased rapidly from day 16 after disease onset (20). D-dimmer levels have also been present at a higher level.
Median high sensitivity troponin I level at 4 days of symptom onset was $8 \mathrm{pg} / \mathrm{mL}$ in non-survivors versus $2.5 \mathrm{pg} / \mathrm{ml}$ in survivors. The levels in survivors did not rise significantly i.e. 2.5 to $4.4 \mathrm{pg} / \mathrm{mL}$ only. However, in case of non-survivors it was found to be as high as $24.7 \mathrm{pg} / \mathrm{mL}$ on day $7,55.7 \mathrm{pg} / \mathrm{mL}$ on day $13,134.5$ $\mathrm{pg} / \mathrm{mL}$ on day 19 and $290.6 \mathrm{pg} / \mathrm{mL}$ on day 22 . Median time of death from symptom onset was found to be 18.5 days (range 15 to 20 days). Thus, rising troponin levels along with increased levels of other markers of inflammation such as D-dimmer, interleukin 6 (IL-6), ferritin, lactate dehydrogenase (LDH) suggests the possibility of cytokine storm or 
secondary hemophagocytic lymphohistiocytosis. Patients presenting with cardiac symptoms are at increased risk of isolated myocardial injury, potentially stress cardiomyopathy or viral myocarditis (21). A case report presented by $\mathrm{Hu}$ et al, showed a man presenting with chest pain and ST segment elevation on electrocardiogram (ECG), left ventricular dysfunction was seen on echocardiogram (ECHO; EF 27\%) and elevated cardiac biomarkers. After treatment with intravenous immunoglobulin and steroids EF improved to $66 \%$ and biomarkers level normalized (21).

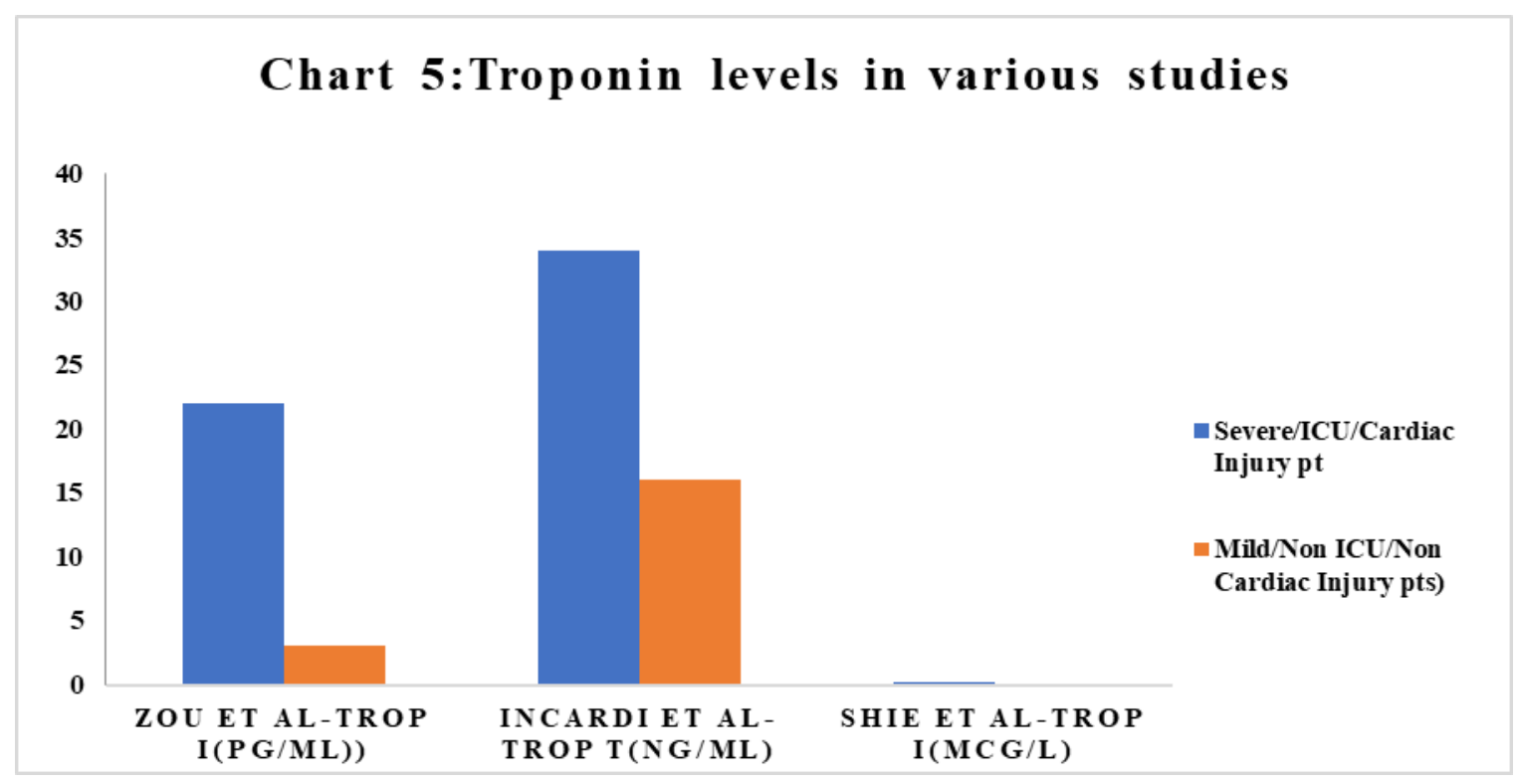

Patients may present with myocardial ischemia, myocarditis, palpitation, breathlessness and even acute heart failure. Heart failure presents with higher rates of mortality as per various studies. Heart failure was the most common cause of death after ARDS, sepsis according to Chen $\mathrm{T}$ et al (22). Zhou et al studies showed that $23 \%$ of the patients and $52 \%$ of the non survivors have heart failure as the complication. (20). Heart failure is one of the major complications in COVID-19 (23). Few patients of COVID-19 have presented with myocarditis and these patients presented with hypotension and low cardiac output even requiring ionotropic supports. On cardiac imaging oedema and diffuse ventricular wall thickening were seen while the biopsy showed inflammatory attributes (24). Myocardial injury occurs not only due to action of the virus per se, but also host of other causes are involved in the pathogenesis. (25). Mediators of atherosclerosis like pro- inflammatory cytokine are responsible for plaque rupture through the inflammatory cascade involving local inflammation, pro coagulant factor stimulation and hemodynamic changes which further lead to ischemia and thrombosis (26).

SARS is a lower respiratory tract disease (27). The point of entry for the SARS-CoV-2 is believed to be via the ACE2 receptors in the lungs and endothelium. ACE2 protein is found in arterial and venous endothelial cells and arterial smooth muscle cells in all of the organs like lungs, small intestine, stomach, colon brain, kidney, nasal and oral mucosa, nasopharynx, skin, muscles around sebaceous glands, basal cell layer of hair follicles and eccrine glands $(28,29)$. Marked presence of ACE2 was seen in type
I and type II alveolar epithelial cells in lungs, small intestine and endothelium which thus serve as a point of entry. The levels of expression of ACE2 in the heart was found to be higher than that in lungs but was comparatively lower than that of intestine and kidney. The reason for increased susceptibility of heart to SARS-CoV-2 could be blamed to higher levels of expression of ACE2 in pericytes of the heart. Patients with heart failure exhibited increased expression of ACE2 and thus are at risk of cardiac manifestation and injury due to the infection (30).

Different organ involvement may also be attributed to the vascular spread of virus. The vascular endothelium is a "hub" for glandular functions like paracrine/endocrine and autocrine features which are important to maintain vascular homeostasis. The viral elements lie within the endothelial cells resulting in inflammatory cell death and thus facilitating the induction of endotheliitis in several organs and host inflammatory response. Endothelial dysfunction shifts the balance towards vasoconstriction resulting in organ ischemia, inflammation with oedema and a pro- coagulant state. It is also important to note that prolonged bed rest may predispose to thromboembolic events (31).

COVID-19 infection results in stimulation of the inflammatory cascade. Increased mediators of inflammation are produced which results in elevation of body temperature manifested clinically by fever. This results in sympathetic activation resulting in tachycardia, increased myocardial oxygen consumption and energy expenditure. This results in increased oxidative stress resulting in formation of 
reactive oxygen species (ROS) leading to acidosis and more commonly mitochondrial damage and less commonly cell death (32).

The pathogenesis is similar to other SARS infection wherein the viral ribonucleic protein (RNA)binds to pattern recognition receptors, the Toll like receptors (TLR) 3,7,8,9 leading to stimulation of the inflammatory cascade resulting in activation of nuclear factor $\kappa \beta(\mathrm{NK}-\kappa \beta)$ and interferon regulatory factor 3(IRF3). This leads to production of pro inflammatory molecules like Interferon $\alpha$, Interferon $\beta$, Interleukins IL 1, IL2, IL4, IL6(the chief among all), IL10, IL12, IFN $\gamma$ and TNF $\alpha$ (Fig. 1). The resultant effects are increased vascular permeability, alveolar epithelial damage leading to ARDS and multiorgan damage (33).
Several mechanisms have been proposed about myocardial damage. Immune dysfunction involving the $\mathrm{T}$ cell and immune signal dysfunction adversely affects the body's response to SARS-CoV-2 infection. Increased expression of proinflammatory cytokines and chemokines especially in the severe cases, the consumption of CD4+ and CD8+ T cells, and the decrease of regulatory $\mathrm{T}$ cells, might result in increased inflammatory responses, production of cytokine storm and make the already damaged tissue even worse (Fig. 1). This was corroborated by the change in laboratory parameters, i.e. elevated chemokine levels, increased NLR and serum cytokine in infected patients. It also correlated with prognosis and outcome, suggesting a possible role for hyperinflammatory responses in COVID-19 pathogenesis (33).

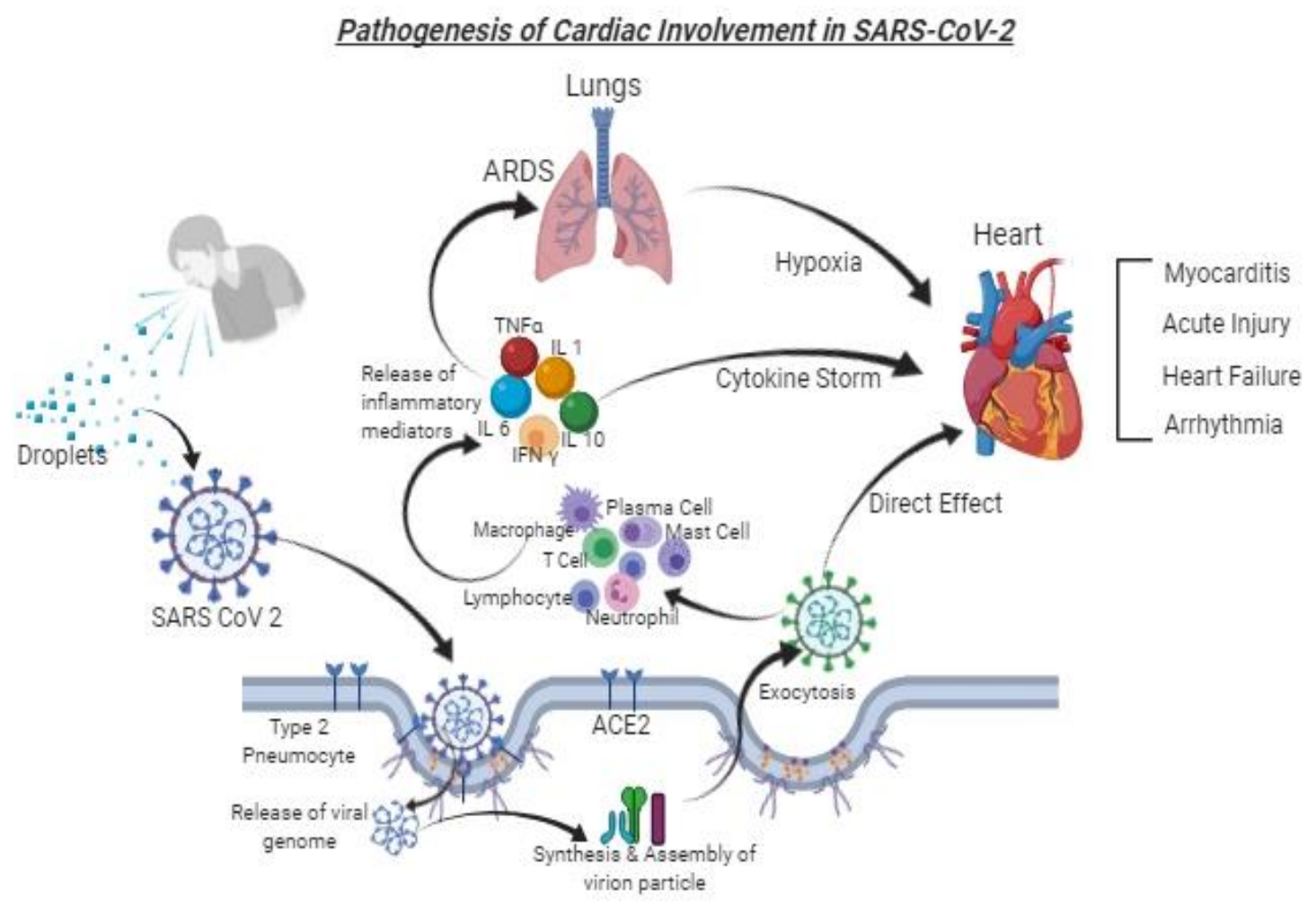

Fig. 1: Pathogenesis cascade

Patients with cardio-vascular co-morbidities like hypertension, diabetes have ineffective adaptation to the increased demand of severe viral illness and decreased systemic oxygenation during the infection (34). This leads to demand-supply imbalance and can result in acute myocardial injury. Increased coronary blood flow can precipitate plaque rupture due to increased shear stress and result in acute myocardial infarction. It can also be precipitated by systemic inflammation as it causes a pro-thrombotic situation (34).

Electrolyte imbalance has been associated with many critical systemic illnesses and can precipitate arrhythmias especially in patients with cardiac disorder. It has been noted that patients with COVID19 were more prone to hypokalaemia and that creatine kinase (CK), CK-MB, lactate dehydrogenase, aspartate transferase, and erythrocyte sedimentation rate were significantly associated with the severity of hypokalaemia $(\mathrm{P}<0.01)$. Collectively, elevation in these values were generally related to myocardial injury. Also, hypokalaemia was most commonly (93\%) seen in severe and critically ill patients and is attributed more to renal loss in comparison to gastrointestinal loss (35).

Previous SARS ancestors were associated with significant cardiac morbidity and mortality (36). Elevated levels of troponin I, NT-proBNP, hs-CRP 
(and also the markers of cardiac injury) correlate with severity of disease. These were also found to be higher among the non-survivors (37). Down regulation of ACE2 impedes the cardio protective effect of angiotensin1-7 thus leading to TNF $\alpha$ over production. Thus, these inflammatory responses may lead to myocardial damage further resulting in cardiac complications like heart failure, age related cardiomyopathy and cardiac dysfunction and thus supporting the claim of severe inflammatory response as a possible mediator of cardiomyocyte damage (38).Along with TNF $\alpha$, SARS virus is believed to induce interstitial fibrosis development in the myocardium through activation of SMAD pathway (similar to development of lung fibrosis) via activation of TGF $\beta$. This could also potentially be a mode of cardiac damage. Exaggerated cytokine response by Type 1 and Type 2 helper $\mathrm{T}$ cells in additional to strong interferon mediated response could also result in myocardial dysfunction (39).
Thus, due to significant resemblance of SARS-CoV infection with COVID-19, possible mechanisms of cardiac injury could include direct damage to cardiomyocyte, severe inflammation, myocardial interstitial fibrosis, interferon mediated immune response, exaggerated cytokine response by Type 1 and 2 helper $\mathrm{T}$ cells, coronary plaque destabilization and hypoxia (39).

\section{The role of ACE2}

ACE2 is an enzyme involved in RAAS pathway. It is a type 1 membrane protein which is primarily involved in the maturation of angiotensin, a peptide hormone that controls vasoconstriction and blood pressure. It is expressed in lungs, heart, kidneys and intestine. It has been noted that decreased expression of ACE2 is associated with cardiovascular diseases. The $\mathrm{N}$ terminal PD of ACE2 cleaves Ang I to produce Ang (1-7). ACE2 can directly process Ang II to give Ang (1-7; 40).

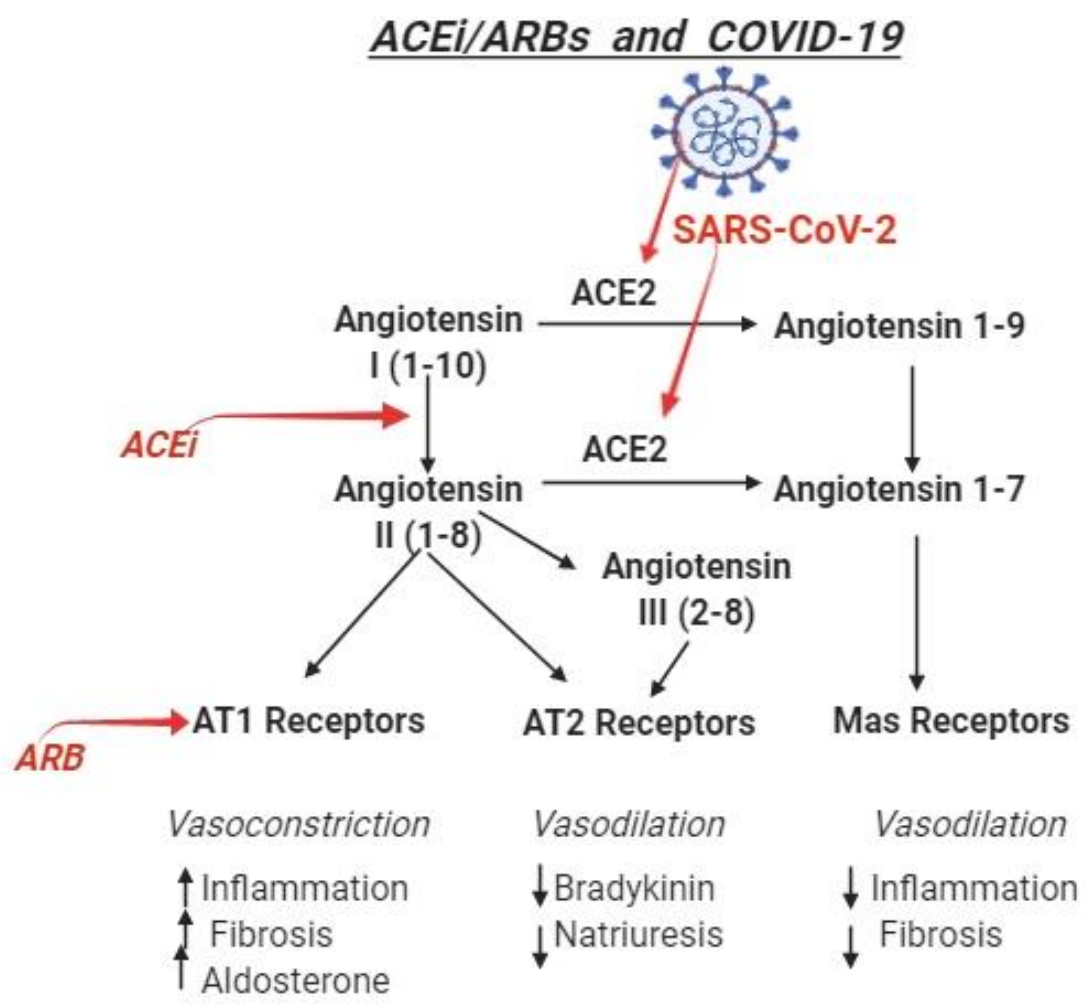

Fig. 2: $\mathrm{ACE}$ and ARBs

Even though it shares a $42 \%$ similarity to ACE, it cannot convert angiotensin I to angiotensin II. Angiotensin II has antagonistic actions to Ang (1 - 7). Contrary to the former, the later has vasodilatory, anti- fibrotic and anti-hypertensive effect (via Mas receptor stimulation; Fig. 2). Ace2 can also convert Angiotensin I to Angiotensin 1-9 (Fig. 2). Ace2 is antagonistic to angiotensin II with vasodilatory, antiinflammatory, antioxidant and anti-fibrotic effects. In animal studies, downregulation of ACE2 results in left ventricular systolic dysfunction and HFrEF. Up regulation in animal studies have resulted in reduction of reactive oxidative stress, fibrosis, and myocardial hypertrophy. It is also believed to play a role in HF with preserved ejection fraction (42). In addition to its cardio protective action, it also has immune protective actions as it favours inflammation by its direct action on macrophages and indirect action by angiotensin II reduction.

ACE2 is upregulated in patients with cardiovascular co morbidities like diabetes and hypertension, and patients on treatment with ACE inhibitors or ARB. (43) Studies by Ziesman et al., have shown the up regulation of ACE2 not only in blood (elevation of Plasma ACE2 levels) but also in cardiac tissue in patients with severe heart failure (44). Studies have 
showed that patients with higher concentrations of ACE2 were more often men, and that they were more likely to have cardiac complications like atrial fibrillation, diabetes, a higher heart rate, and a lower systolic blood pressure (45). Studies by $\mathrm{Wu}$ et al., and Sama et al., confirmed the same (46). Thus, increased ACE2 in patients with cardiac complication increases their susceptibility as well infectivity to SARS-CoV-2(94-98). However increased susceptibility of patients on ACEi/ARBs to COVID19 has not been proven.

Wrapp et al., showed the biophysical and structural evidence that the 2019-nCoV S protein binds angiotensin-converting enzyme 2 (ACE2) with higher affinity than does severe acute respiratory syndrome (SARS)-CoV S protein (47). The SARS-CoV-2 Receptor Binding Motiff contains structural changes in the ACE2 binding ridge largely caused by a fourresidue motif. This allows the ridge to be more compact and forms better contact with $\mathrm{N}$ terminal helix of ACE2 in comparison to SARS-CoV. Another structural advantage over its predecessor is the presence of furin cleavage site which increases its infectivity potential. The spike glycoprotein $S$ of the virus is cleaved into S1 and S2. Binding of ACE2 occurs at $\mathrm{S} 1$ subunit of the virus. Once binding is complete, there is fusion of the membrane and invagination of the virus into the host cell. The spike protein also causes activation of ADAM-17/TACE, which cleaves and releases ACE2, endocytosis of the ligand/receptor complex followed by intracellular digestion resulting in downregulation of ACE2.

\section{ACEi/ARB treatment and COVID-19}

Numerous studies are being conducted in order to establish the overall effect of ACEi/ARBs in COVID-19. Studies have shown that the levels of ACE2 are higher in patients on ACEi/ARB treatment, thus increasing their susceptibility to SARS-CoV-2 and that patients on ACEi/ARBs, have decreased effect of Angiotensin II via AT1 receptors. Reports support the fact that there is no need to stop these medications in patients with COVID-19 (48). There has been an inconsistent effect of ACEi/ARBs on the levels of plasma ACE2. None of the studies have been able to demonstrate effects of ACEi/ARBs on lung specific ACE2 (as mode of entry for the virus is respiratory epithelium). Also, most of the patients with cardiac complications have been on beta blockers which itself has a negative effect on ACE2 activity.

Studies by Zhang et al., showed that patients who received ACEi/ARBs had a lower risk of COVID-19 infection (adjusted hazard ratio, 0.37 [95\% CI, 0.150.89 ]; $P=0.03$ ) and lower rate of mortality (adjusted hazard ratio, 0.30 [95\% CI, 0.12-0.70]; $P=0.01$ ) in patients with COVID-19 and coexisting hypertension. No increased risk of in-hospital death was found to be associated with the use of ACE inhibitors as per Mehra et al., (14). A populationbased case control study from Italy also concluded that ACEi/ARBs were not an independent predictor of SARS-CoV-2 or the severity of the infection. Similar results were obtained from Inciardi et al study as well (16).

\section{Heart transplantation and COVID-19}

A case study by Li and Dong described two heart transplant recipients during this COVID-19 period. One presented with mild disease and the other had severe symptoms requiring oxygenation. Computerized tomography (CT) of lung showed bilateral ground glass opacities in a peripheral distribution. Both were treated with antibiotic and antiviral agents and managed to survive. The patient with severe symptoms required stoppage of immunosuppressive medication followed by treatment with methyl prednisolone and intravenous immunoglobulin. A study by Ren et al., in 87 heart transplants recipients showed recipients who followed strict precautionary measures had an uneventful post-transplant period (49).

People with cardiovascular risk factors like hypertension, diabetes, heart failure are at a higher risk of infection and increased severity of corona virus disease manifestations. Cardiac injury can be measured with the help of biomarkers like troponins, NT-proBNP and hs-CRP and so it is importance to check these values in patients who are at increased risk or are having severe symptoms as it can predispose the patients to complications e.g. arrhythmias, heart failure or even shock (50).

\section{CONCLUSION}

There is a higher risk of co morbidly in COVID-19 infection. Role of ACEi/ARB in SARS-CoV-2 are not known in great detail. However, the available literature doesn't advise the discontinuation of these drugs in the patients and there is a subtle hint that these drugs may be beneficial in these patients. It is very important to prevent complications of cardiac injury and it must be recognized at an earlier stage itself. Increased complications result in increased duration of hospital stay, decreased quality of life, increased ambulatory care and no doubt a bigger financial burden not only for oneself but on the community as well.

\section{CONFLICT OF INTEREST}

Authors declare no conflict of interest

\section{REFERENCES}

1. Virchow, R. Die Cellularpathologie in Ihrer Begründung auf Physiologische und Pathologische Gewebelehre. Hirschwald; 1858.

2. Mann, D. L., Chakinala, M. Heart Failure: Pathophysiology and Diagnosis; Harrisons Principles of Internal Medicine; $20^{\text {th }}$ edition. $1763-1768$. 
3. Kuiken, T., Fouchier, R. A. M., Schutten, M., Rimmelzwaan, G. F., Amerongen, G., Riel, D., et al., Newly discovered corona virus as the primary cause of severe acute respiratory syndrome. Lancet 2003; 362: 263-270.

4. De Groot, R. J., Baker, S. C., Baric, R. S., Brown, C. S., Drosten, C., Enjuanes, L., et al., Middle East respiratory syndrome corona virus (MERS-CoV): announcement of the Corona virus Study Group. J Virol. 2013; 87: 7790-7792.

5. World Health Organization (WHO) Emergency Committee. Statement on the second meeting of the International Health Regulations (2005) Emergency Committee regarding the outbreak of novel corona virus (2019-nCoV). Geneva: WHO; 30 January 2020.

6. WHO situation reports 209 https://www.who.int/docs /defaultsource/coronaviruse/situation-reports/20200816covid-19sitrep209.pdf?sfvrsn=5dde1ca 2_2

7. WHO report. Transmission of SARS-CoV-2. implications for infection prevention precautions; Scientific Brief; 09/07/2020.

8. Zheng, Y. Y., Ma, Y. T., Zhang, J. Y., and Xie, X. COVID19 and the cardiovascular system. Nat Rev Cardiol. 2020, 10.1038/s41569-020-0360-5.

9. Xiong, T. Y., Redwood, S., Prendergast, B., Chen, M. Coronaviruses and the cardiovascular system: acute and long-term implications. Eur Heart J. 2020, 10.1093/eurheartj/ehaa231.

10. Wu, C., Chen, X., Cai, Y., Xia, J., Zhou, X., Xu, S., et al., Risk factors associated with acute respiratory distress syndrome and death in patients with corona virus disease 2019 pneumonia in Wuhan, China. JAMA Intern Med. 2020, 10.1001/jamainternmed.2020.0994.

11. Yang, J., Zheng, Y., Gou, X., Pu, K., Chen, Z., Guo, Q., et al., Prevalence of co morbidities and its effects in corona virus disease 2019 patients: A systematic review and metaanalysis. Int J Infect Dis. 2020; 94: 91-95.

12. Arentz, M. Y. E., Klaff, L., Lokhandwala, S., Riedo, F. X., Chong, M., Lee, M. Characteristics and Outcomes of 21 Critically Ill Patients With COVID-19 in Washington State. JAMA. 2020, 10.1001/jama.2020.4326.

13. Wang, D., Hu, B., Hu, C., Zhu, F., Liu, X., Zhang, J., et al., Clinical Characteristics of 138 Hospitalized Patients with 2019 Novel Corona virus-Infected Pneumonia in Wuhan, China. JAMA. 2020, 10.1001/jama.2020.1585.

14. Mehra, M. R., Desai, S. S., Kuy, S., Henry, T. D., Patel, A. N. Cardiovascular Disease, Drug Therapy, and Mortality in COVID-19. N Engl J Med. 2020, 10.1056/NEJMoa2007621.

15. Mo, P., Xing, Y., Xiao, Y., Deng, L., Zhao, Q., Wang, H., et al., Clinical characteristics of refractory COVID-19 pneumonia in Wuhan,China. Clin Infect Dis. 2020, 10.1093/cid/ciaa270.

16. Inciardi, R. M., Adamo, M., Lupi, L., Cani, D. S., Di Pasquale, M., Tomasoni, D., Italia, L., et al. Characteristics and outcomes of patients hospitalized for COVID-19 and cardiac disease in Northern Italy. European heart journal. 2020 May 14;41(19):1821-1829.

17. Klein, S. L., Flanagan, K. L. Sex differences in immune responses. Nat Rev Immunol. 2016; 16: 626-638.

18. Wu, C., Chen, X., Cai, Y., Xia, J., Zhou, X., Xu, S., et al., Risk factors associated with acute respiratory distress syndrome and death in patients with coronavirus disease 2019 pneumonia in Wuhan, China. JAMA internal medicine. 2020 Mar 13

19. Huang, C., Wang, Y., Li, X., Ren, L., Zhao, J., Hu, Y., et al., Clinical features of patients infected with 2019 novel coronavirus in Wuhan, China. The lancet. 2020 Feb 15;395(10223): 497-506.

20. Zhou, F., Yu, T., Du, R., Fan, G., Liu, Y., Liu, Z., et al., Clinical course and risk factors for mortality of adult in patients with COVID-19 in Wuhan, China: a retrospective cohort study; VOLUME 395, ISSUE 10229, P1054-1062, MARCH 28, 2020; The Lancet.
21. Hu, H., Ma, F., Wei, X., Fang, Y. Coronavirus fulminant myocarditis treated with glucocorticoid and human immunoglobulin. European heart journal. 2020 Mar 16.

22. Chen, T., Wu, D., Chen, H., Yan, W., Yang, D., Chen, G., et al., Clinical characteristics of 113 deceased patients with corona virus disease 2019: retrospective study. BMJ. 2020; 368:m1091.

23. Dong, N., Cai, J., Zhou, Y., Liu, J., Li, F. End-stage Heart Failure with COVID-19: Strong Evidence of Myocardial Injury by 2019-nCoV. JACC Heart Fail. 2020, 10.1016/j.jchf.2020.04.001.

24. Inciardi, R. M., Lupi, L., Zaccone, G., Italia, L., Raffo, M., Tomasoni, D., et al., Cardiac Involvement in a patient with Corona virus Disease 2019 (COVID-19). JAMA Cardiology. 2020, 10.1001/jamacardio.2020.1096.

25. Xu, Z., Shi, L., Wang, Y., Zhang, J., Huang, L., Zhang, C., et al., Pathological findings of COVID-19 associated with acute respiratory distress syndrome. Lancet Respir Med. 2020; 8: 420-422.

26. Zhou, R. Does SARS-CoV-2 cause viral myocarditis in COVID-19 patients? Eur Heart J. 2020, 10.1093/eurheartj/ehaa392.

27. Ding, Y., Wang, H., Shen, H., Li, Z., Geng, J., Han, H., et al., The clinical pathology of severe acute respiratory syndrome (SARS): a report from China. J Pathol 2003; 200: 282-289.

28. Hamming, I., Timens, W., Bulthuis, M. L. C., Lely, A. T., Navis, G. J., Goor, H. Tissue distribution of ACE2 protein, the functional receptor for SARS corona virus. A first step in understanding SARS pathogenesis; J Pathol. 2004 Jun; 203(2): 631-637.

29. Chen, L., Li, X., Chen, M., Feng, Y., Xiong, C. The ACE2 expression in human heart indicates new potential mechanism of heart injury among patients infected with SARS-CoV-2. Cardiovasc Res. 2020; 116: 1097-1100.

30. Flammer, A. J., Anderson, T., Celermajer, D. S. The assessment of endothelial function: from research into clinical practice. Circulation. 2012; 126: 753-767.

31. Klok, F. A., Kruip, M., Meer, N. J. M., Arbus, M. S., Gommers, D.A.M.P.J., et al., Incidence of thrombotic complications in critically ill ICU patients with COVID-19. Thromb Res. 2020, 10.1016/j.thromres.2020.04.013.)

32. Takasu, O., Gaut, J. P., Watanabe, E., To, K., Fagley, R. E., Sato, B., et al., Mechanisms of cardiac and renal dysfunction in patients dying of sepsis. Am J Respir Crit Care Med. 2013; 187: 509-517.

33. Qin, C., Zhou, L., Hu, Z., Zhang, S., Yang, S., Tao, Y., et al., Dysregulation of immune response in patients with COVID-19 in Wuhan, China. Clin. Infect. Dis. 2020 doi: 10.1093/cid/ciaa248.

34. Adão, R. Inside the heart of COVID-19. Cardiovasc. Res. 2020 doi: 10.1093/cvr/cvaa086.

35. Chen, D., Li, X., Song, Q., Hu, C., Su, F., Dai, J. Hypokalemia and clinical implications in patients with corona virus disease 2019 (COVID-19) medRxiv. 2020 doi: 10.1101/2020.02.27.20028530.

36. Li, S. S., Cheng, C. W., Fu, C. L., Chan, Y. H., Lee, M. P., Chan, J. W. Left ventricular performance in patients with severe acute respiratory syndrome: a 30-day echocardiographic follow-up study. Circulation. 2003; 108: 1798-1803.

37. Chen, C., Jiangtao, Y., Ning, Z., Jianping, Z., Daowen, W. Analysis of myocardial injury in patients with COVID-19 and association between concomitant cardiovascular diseases and severity of COVID-19. Chinese J Cardiol. 2020; 48: E008

38. Cameron, M. J., Bermejo-Martin, J. F., Danesh, A., Muller, M. P., Kelvin, D. J. Human immunopathogenesis of severe acute respiratory syndrome (SARS) Virus Res. 2008; 133: 13-19.

39. Wong, C. K., Lam, C. W., Wu, A. K., Lp, W. K., Lee, N. L. S., et al., Plasma inflammatory cytokines and chemokines in 
severe acute respiratory syndrome. Clin. Exp. Immunol. 2004; 136: 95-103.

40. Babapoor-Farrokhran, S., Gill, D., Walker, J., Rakekhi, R., Bozorgnia, B., Amanullah, A. Myocardial injury and COVID-19: Possible mechanisms; Life Sci. 2020 Jul 15; 253: 117723; Published online 2020 Apr 28. doi: 10.1016/j.lfs.2020.117723

41. Donoghue, M., Hsieh, F., Baronas, E., Godbut, K., Gosselin, M., Stagliano, N., et al., A novel angiotensin-converting enzyme-related carboxypeptidase (ACE2) converts angiotensin I to angiotensin 1-9. Circ. Res. 87, E1-E9 (2000). 10.1161/01.RES.87.5. e1.

42. Zhao, Y., Zhao, Z., Wang, Y., Zhou, Y., Ma, Y., Zuo, W. Single-cell RNA expression profiling of ACE2, the putative receptor of Wuhan 2019-nCov. bioRxiv 2020.0126.919985 [Preprint]. $26 \quad$ January 2020. 10.1101/2020.01.26.919985.10.1101/2020.01.26.919985.

43. Zisman, L. S., Keller, R. S., Weaver, B., Lin, Q., Speth, R., Bristow, M. R. Increased angiotensin-(1-7)-forming activity in failing human heart ventricles: Evidence for upregulation of the angiotensin-converting enzyme homologue ACE2. Circulation 108, 1707-1712 (2003). 10.1161/01.CIR.0000094734.67990.99.

44. Raizada, M. K., Ferreira, A. J. ACE2: A new target for cardiovascular disease therapeutics. J. Cardiovasc. Pharmacol. 2007; 50: 112-119. 10.1097/FJC.0b013e3180986219.

45. Epelman, S., Tang, W. H., Chen, S. Y., Lente, F., Francis, G. S., Sen, S. Detection of soluble angiotensin converting enzyme 2 in heart failure: insights into the endogenous counter-regulatory pathway of the renin-angiotensinaldosterone system. J Am Coll Cardiol. 2008; 52: 750-754.

46. Wu, Z., and Mc-Googan, J. M. Characteristics of and Important Lessons from the Coronavirus Disease 2019 (COVID-19) Outbreak in China: Summary of a Report of 72314 Cases from the Chinese Centre for Disease Control and Prevention. JAMA. 2020, 10.1001/jama.2020.2648.

47. Wrapp, D., Wang, N., Corbett, K. S., Goldsmith, J. A., Hsieh, C- L., Abiona, O., Graham, B. Cryo-EM structure of the 2019-nCoV spike in the prefusion conformation. Science eabb2507 (2020). 10.1126/science. abb2507.

48. Zhang, P., Zhu, L., Cai, J., Lei, F., Qin, J., Xie, J., et al., Association of Inpatient Use of Angiotensin-Converting Enzyme Inhibitors and Angiotensin II Receptor Blockers with Mortality Among Patients with Hypertension Hospitalized With COVID-19; Circ Res. 2020 Jun 5; 126(12): 1671-1681. Published online 2020 Apr 17. doi: 10.1161/CIRCRESAHA.120.317134.

49. Ren, Z., Hu, R., Wang, Z., Zhang, M., Ruan., Y., Wu, Z., et al., Epidemiologic and clinical characteristics of heart transplant recipients during the 2019 corona virus outbreak in Wuhan, China: A descriptive survey report; J Heart Lung Transplant; 2020 May; 39(5): 412-417; doi: 10.1016/j.healun.2020.03.008. Epub 2020 Mar 25.

50. Tomasoni D., Italia, L., Adamo, M., Inciardi, R., Lombardi, C.M., Solomon, S.D., et al. COVID-19 and heart failure: from infection to inflammation and angiotensin II stimulation. Searching for evidence from a new disease; Eur J Heart Fail; 2020 May 15;10.1002/ejhf.1871; doi: 10.1002/ejhf.1871. 